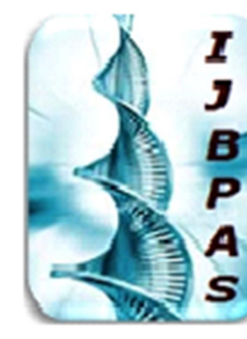

International Journal of Biology, Pharmacy and Allied Sciences (IJBPAS)

'A Bridge Betuen Caboratory and QRendo'

Www.ibpas.com

\title{
MAJOR LOWER-LIMB AMPUTATION DURING THE COVID PANDEMIC IN INDIA, DUE TO COMPLICATIONS OF COVID 19 LIKE THROMBUS FORMATION
}

\section{MADHWAPATHY NS ${ }^{1}$, JANUGADE HB ${ }^{2}$ AND GOUDA $S^{3}$}

1. Junior Resident, Department of Surgery, Krishna Institute of Medical Sciences Deemed To Be University, Karad, Maharashtra (India)

2. Professor, Department of Surgery, Krishna Institute of Medical Sciences Deemed To Be University, Karad, Maharashtra (India)

3. Senior Resident, Department of Surgery, Krishna Institute of Medical Sciences Deemed To Be University, Karad, Maharashtra (India)

*Corresponding Author: Dr. Nandan S.Madhwapathy:

Received 12 ${ }^{\text {th }}$ July 2021; Revised $14^{\text {th }}$ Aug. 2021; Accepted $27^{\text {th }}$ Oct. 2021; Available online $15^{\text {th }}$ Feb. 2022

https://doi.org/10.31032/IJBPAS/2022/11.2.1017

ABSTRACT

Previous or current COVID 19 infections are more likely to have lower limb amputations than patients without previous or current COVID 19 infections. The risk of preventable lower limb amputations has increased during the 2019 coronavirus disease (COVID-19) lockdown period. Therefore, we conducted a single-center retrospective study of major amputations during the prepandemic period (March 25, 2019 to December 31, 2019) and the pandemic period (March 25, 2020 to December 31, 2019). 2020). During the pre-pandemic period, 24 lower limb amputations (below and above the knee amputations) were performed and 37 major amputations were performed during the pandemic period. There was a $54.1 \%$ increase in major amputations during the pandemic period rather than the pre-pandemic period. This study demonstrates the indirect effect of the COVID-19 pandemic on people with co-morbidities, leading to a higher incidence of lower limb amputations (amputations below and above the knee) which can have a drastic impact on their quality of life. This study demonstrates the increased risk of thrombus formation as a long-term complication of COVID 19. 


\section{INTRODUCTION}

The 2019 coronavirus disease (COVID-19) pandemic has forced a global shutdown. In India, there was strict containment from March 2020 to November 2020 to contain the spread of the disease. Only essential services were open and all other categories were closed during the period of strict containment. This made it difficult for people with ulcers and gangrenic changes to see their doctor regularly. People who developed diabetic foot infections were also unable to see their surgeon for procedures such as debridement. This can lead to many preventable lower limb amputations. A significant increase in complications of diabetic foot. The aim of this study was to find out the changes in the amputation rate in people with diabetes during the COVID-19 pandemic in India. Therefore, we conducted a retrospective study focusing on the main amputation events during the pre-pandemic (March 25, 2019 to December 31, 2019) and pandemic (March 25, 2020 to December 31, 2020) period.

\section{Criteria for sample selection:}

Inclusion criteria:

All COVID-19 positive status patients with CT/USG DOPPLER diagnosed acute limb ischemia.

\section{Exclusion criteria:}

1. Patients who are unwilling to participate

2. Patients with peripheral vascular disease

\section{METHODS AND MATERIALS}

For study participants in the pandemic and pre-pandemic groups, hospital identification numbers and date of surgery were taken from occupational therapist records. Subsequently, the identification numbers were used to retrieve the other parameters from the KIMS HOSPITAL database for Diabetes. Large amplifications recorded from March 25, 2019 to December 31, 2019 were considered as the pre-pandemic period and large amplifications from March 25, 2020 to December 31, 2020 were considered as the pandemic period according to the lockout notice in India. the study included a previous or current CVID 19 infection, over 18 years of age, who visited KIMS Hospital for ulcers. Age, gender, length of diabetes, comorbidities, complications, type of amplification (below the knee - BK or above the knee - AK), type of medication, hospitalization, level of glycosylated hemoglobin $(\mathrm{HbA} 1 \mathrm{c})$, blood pressure, and history of surgery (collected if applicable). The hypothesis of this retrospective, singlecenter study was the increased number of large amplifications in people with diabetes 
in the pandemic period compared to the pre-pandemic period. 5,351 and 3,591 people with diabetes received inpatient treatment at KIMS Hospital for peptic ulcer disease in the pre-pandemic and pandemic periods.

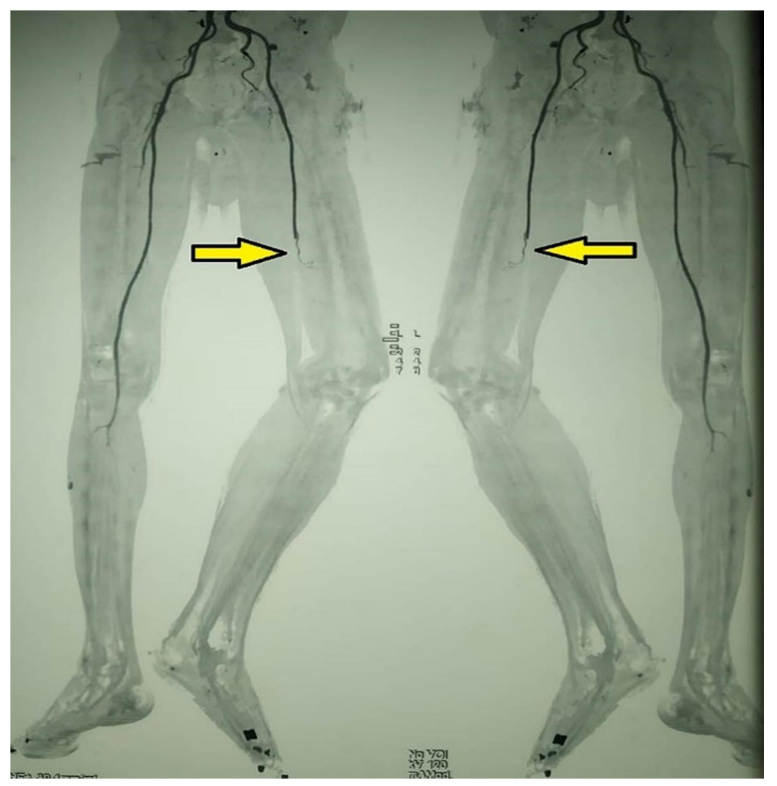

\section{Statistical analysts}

The data collected was entered into Microsoft Excel and the variables were coded. It was exported to IBM SPSS. Descriptive analysis was used to illustrate the data. The mean and standard deviation were used to represent hospitalization, age of study participants, length of diabetes of study participants, pulley length, blood pressure (systolic and diastolic), glycosylated hemoglobin, CT or Doppler angiography results, and hemoglobin levels. Time of entry. Frequencies and percentages were used to indicate other parameters, including BK amplification number, AK amplifications, gender, presence of complications, and previous surgery history. Wilcoxon's signature ranking test was used because the data were not normal. IBM version 20 SPSS was used.

\section{DISCUSSION}

The majority of the study participants were male. This resembles the study conducted by Caruso et al [2], which indicated increased diabetic foot problems during the pandemic in tertiary care settings. Among major amputations, BK amputations were higher in number than $\mathrm{AK}$ in both the pandemic and prepandemic groups. The overall prevalence of major amputations was higher during the pandemic lockdown compared to the prepandemic period. There was a $54.1 \%$ increase in major amputations 
noted. This might be due to the increased severity ulcer with thrombus in the pandemic period [3]. The reason might be the outcome of postponing the routine visit, improper diet, nonadherence to medications, and physical inactivity. Missed hospital visits or postponing the routine visit in this contest is defined as not visiting the physician as per the schedule, which is usually discussed in their previous visits as per the need/risk of the patient. High-risk patients are usually advised to visit their diabetologist and foot care specialists every 3 months. Improper diet can be defined as inconstant dietary habits in opposition to the advice of their physician/dietitian.

In an online pilot survey, $20 \%$ of the study participants recorded they were not following the diet as before the lockdown [4]. Nonadherence to medications is not to take medicines as prescribed by the doctor. This nonadherence can be intentional or nonintentional. People with diabetes are advised to do minimal physical activity (30 min per day) in any form including walking, exercises, and yoga. Not doing the minimal physical activity to control the glycemic index is considered as physical inactivity. In terms of risk factors for diabetes-related amputations, glycemic control, peripheral neuropathy, and PAD are generally considered to play a vital role
$[5,6]$. In this study, the HbAlc of the pandemic group was significantly higher than the prepandemic group (even though it is not statistically significant). A higher prevalence of PAD and peripheral neuropathy prevalence was noted in the study participants in the pandemic group than the prepandemic group. Practices like telemedicine were also practiced in this pandemic globally [7]. In addition to the teleconsultation, home visits by the podiatrists and/or the other paramedical staff were practiced [7]. This combination of treatment in treating the wound can save the limbs and avoid the patients' rush to emergency care at the 11th hour. Even though these services were available, they might not be affordable for all the patients who seek these services due to their socioeconomic status, especially in a developing country like India. During this pandemic, the low socioeconomic groups' routine income was affected [8]. This might have worsened the condition.

\section{RESULTS}

In the preclinical phase, 24 major amplifications were performed (BK, 17 and $\mathrm{AK}, 7)$, and in the pandemic phase, 37 major amplifications were performed (BK, 30 and $A K, 7)(P=0.527)$. The ages of study participants in the pre-pandemic and pandemic periods with large amplifications were $61 \pm 13.4$ and $63.88 \pm 11.08$ years, 
respectively $(\mathrm{P}=0.241)$. The majority of study participants in both time periods were male (pre-pandemic, $\mathrm{M}: \mathrm{F}=20: 4$; pandemic, $\mathrm{M}: \mathrm{F}=29: 8)(\mathrm{P}=0.705)$. The pulley duration of the study participants in the pre-pandemic group was $12.3 \pm 6.6$ years and $14.06 \pm 7.48(\mathrm{P}=0.822)$ years in the pandemic group. Average HbA1c levels in the pandemic group were relatively higher $(9.35 \pm 2.39)$ in the study participants than in the pre-pandemic group, possibly due to the decrease in hospital visits due to locking $(8.88 \pm 2.67)$ $(\mathrm{P}=0.391)$. Clinical signs were also lower in the pandemic group $(52.21 \pm 27.05)$ than in the pre-pandemic group $(61.41 \pm 26.68)$ $(\mathrm{P}=0.040)$. There was no significant change in hospitalization during the prepandemic period $(9.3 \pm 2.9)$ and admissions during the pandemic period $(9.1 \pm 4)(\mathrm{P}=$ $0.851)$. The mean duration of the pulley in the pre-pandemic period was 9 days and the same duration during the pandemic period was 10.8 days $(P=0.269)$. Previous history of foot surgery was noted in 2 of the study participants in the prepandemic group, compared with 9 participants in the pandemic group $(\mathrm{P}=0.655)$

Peripheral neuropathy was present in 21 study participants during the prepandemic period and in 33 participants during the pandemic period $(\mathrm{P}=1000)$. Diabetic nephropathy was observed in 7 study participants in the prepandemic group and 13 participants in the pandemic group $(\mathrm{P}=$ 0.705). Peripheral arterial disease (PAD) was present in 14 study participants in the prepandemic group and 22 in the pandemic group $(\mathrm{P}=1,000)$. Hypertension was present in 13 and 24 study participants in the prepandemic and pandemic groups, respectively $(\mathrm{P}=0.248)$. The amplification rate in the prepandemic and pandemic phases was 4.48 per 1000 and 10.30 per 1000 , respectively.

\section{Acknowledgment}

I am thankful to Mrs Rupali Salunkhe for secretariat help, Krishna institute of medical sciences Deemed to be Unitversity, Karad, Maharashtra (India)

\section{REFERENCES}

[1] A Atri A, Kocherlakota CM, Dasgupta $\mathrm{R}$, Managing diabetic foot in times of COVID-19: time to put the best 'foot' forward. International journal of diabetes in developing countries, 2020, 1-8.

[2] Caruso P, Longo M, Signoriello S, Caruso P, Longo M, Signoriello S, et al, Diabetic foot problems during the COVID-19 pandemic in a tertiary care center: the emergency among the emergencies, Diabetes care, 43(10), 2020, e123-4.

[3] Lancaster EM, Wu B, Iannuzzi J, Rastogi A, Hiteshi P, Bhansali AA, et 
$a l$, Virtual triage and outcomes of diabetic foot complications during Covid-19 pandemic: A retroprospective, observational cohort study, Plos one, 16(5), 2021, e0251143.

[4] Nachimuthu S, Vijayalakshmi R, Sudha M, Viswanathan V, Coping with diabetes during the COVID-19 lockdown in India: results of an online pilot survey, Diabetes \& Metabolic Syndrome: Clinical Research \& Reviews, 14(4), 2020, 579-82.

[5] Verrone Quilici MT, Del Fiol FD, Franzin Vieira AE, Toledo MI, Risk factors for foot amputation in patients hospitalized for diabetic foot infection, Journal of diabetes research, 2016.

[6] LeLevi M, Thachil J, Iba T, Levy JH, Coagulation abnormalities and thrombosis in patients with COVID-19, The Lancet Haematology, 7(6), 2020, e438-40.

[7] Levi M, Thachil J, Iba T, Levy JH, Coagulation abnormalities and thrombosis in patients with COVID-19, The Lancet Haematology, 7(6), 2020, e438-40.

[8] Sypniewska G. Pro-inflammatory and prothrombotic factors and metabolic syndrome, EJIFCC, 18(1), 2007, 39.

[9] Anwar S, Acharya S, Shabih S, Khabut A, Acute limb ischemia in COVID-19 disease: a mysterious coagulopathy, Cureus, 12(7), 2020.

[10] Klok FA, Kruip MJ, Van der Meer NJ, Arbous MS, Gommers DA, Kant KM, et al, Incidence of thrombotic complications in critically ill ICU patients with COVID-19, Thrombosis research, 191, 2020, 145-7.

[11] Helms J, Tacquard C, Severac F, Leonard-Lorant I, Ohana M, Delabranche $\mathrm{X}$, et al, High risk of thrombosis in patients with severe SARS-CoV-2 infection: a multicenter prospective cohort study, Intensive care medicine, 46(6), 2020, 1089-98. 